\title{
ATTENUATION MEASUREMENTS OF SPLIT NORMAL MODES FOR THE 1960 CHILEAN AND 1964 ALASKAN EARTHQUAKES
}

\author{
By Seth Stein and Robert J. Geller
}

\begin{abstract}
Measurements of attenuation for the Earth's longest period modes can be significantly biased by the effects of frequency splitting. Using our previously developed methods of time domain synthesis of split normal modes, we measure $Q$ without such a bias. We also conduct numerical experiments to confirm the errors in $Q$ measurements which result from neglecting the effects of splitting. In contrast to frequency domain this time domain technique allows us to reject data below the ambient noise level for each mode. The $Q$ 's of the longest period spheroidal $\left({ }_{0} S_{2}-0 S_{5}\right)$ and torsional $\left({ }_{0} T_{3}-0 T_{4}\right)$ modes are determined using long $(500 \mathrm{hr})$ records from the Chilean and Alaskan earthquakes.
\end{abstract}

\section{INTRODUCTION}

Measurements of attenuation were among the earliest analyses conducted on the normal mode data for the 1960 Chilean earthquake. The primary focus of interest was on the longest period modes with periods greater than $20 \mathrm{~min}$. Benioff et al. (1961) used spectral widths to measure the $Q$ of ${ }_{0} S_{3}$ from the Isabella record. Alsop et al. (1961a) used a time decay method to find the $Q$ 's of the low-order spheroidal modes, and Ness et al. (1961) estimated the $Q$ of ${ }_{0} S_{0}$. Similar analyses were conducted on the data from the 1964 Alaskan earthquake. Smith (1972) summarized all longperiod $Q$ results to that date, including those of Slichter (1967).

Additional studies of long-period $Q$ have recently been conducted by Sailor and Dziewonski (1978) and by Buland and Gilbert (1978). This renewed interest is motivated by the need to correct earth models for the effects of physical dispersion caused by anelasticity (Akopyan et al., 1975, 1976; Liu et al., 1976), and a desire to derive models of the $Q$ structure of the Earth.

Attenuation measurements for the longest period modes are complicated by frequency splitting (Ness et al., 1961; Benioff et al., 1961). Pekeris et al. (1961) and Backus and Gilbert (1961) showed that the splitting could be explained by the Earth's rotation. The $2 l+1$ singlets in the multiplet of angular order $l$ are split such that each one has a distinct eigenfrequency, amplitude, and phase. The individual singlets are also broadened by attenuation. Thus the splitting is observable only for low-angular order (long-period) multiplets for which the frequency separation due to splitting of the singlets is greater than the spectral line broadening due to attenuation. The combined effects of splitting and peak broadening due to attenuation pose substantial difficulty for $Q$ measurements.

For a single damped harmonic oscillator, attenuation can be easily measured in either the time or the frequency domain. In the time domain, the amplitude is given by

$$
A(t)=e^{t[-\omega / 2 Q+i \omega]}
$$

and $Q$ can be found from the ratio of the amplitudes at two different times. Equivalently, $Q$ can be determined from the width of the spectral peak by using

$$
Q=\omega / \Delta \omega
$$


However, for split modes, it is not possible to derive a simple expression for the interference, either in the time or frequency domains, which will isolate the terms involving $Q$. Thus, to measure $Q$, it is necessary to know the amplitude and phase of each singlet, and to combine them to form a time series including the interference effects. Alsop et $a l$. (1961b) proposed conditions under which $Q$ might successfully be measured, despite the splitting. The time-domain $Q$ measurement technique used in this paper is valid without any such limiting assumptions. Also, in contrast to frequency-domain techniques, time-domain $Q$ measurements allow us to reject data below the ambient noise level for each mode.

\section{Time Domain Synthesis Method}

Our attenuation measurement method is based on the results of Stein and Geller (1977) for the theoretical amplitude and phase of the singlets excited by an earthquake source: a double couple of arbitrary orientation resulting from slip on a fault plane. Figure 1, from Geller and Stein (1977), shows the spectra of the spheroidal
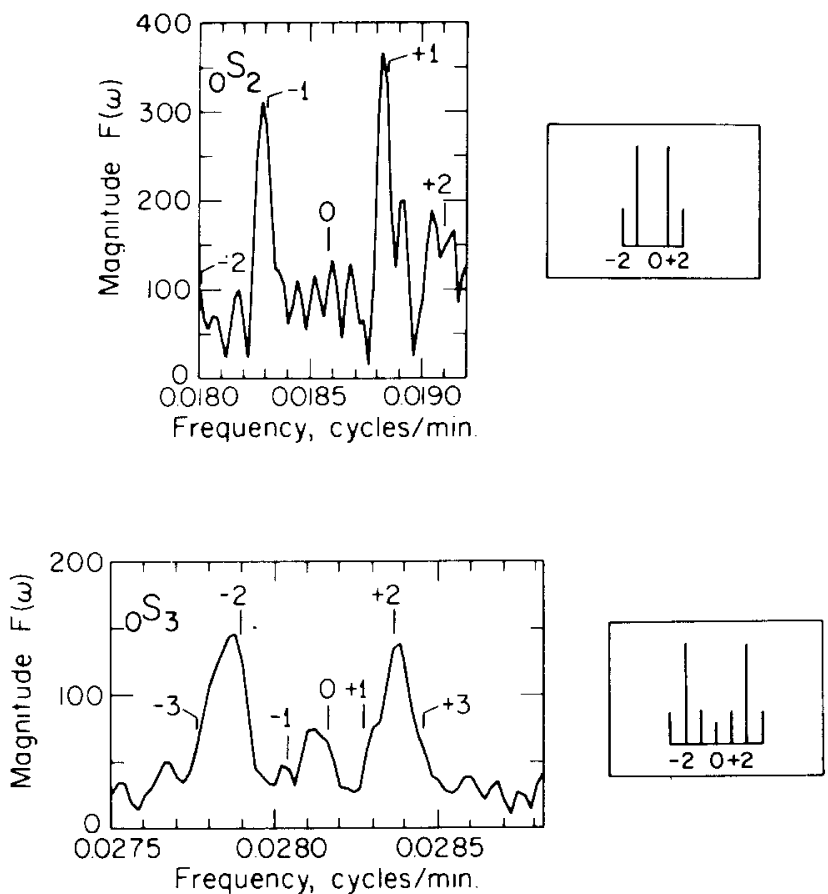

Fig. 1. Split spheroidal mode spectra for ${ }_{0} S_{2}(t o p)$ and ${ }_{0} S_{3}$ (bottom) excited by the Chilean earthquake, as observed on a strainmeter at Isabella, California. The Eigenfrequency separation is taken from Dahlen (1968), but the central frequency has been chosen to yield a best fit with the observed peaks. Synthetic relative spectra for the finite fault geometry of Kanamori and Cipar (1974) are given for each mode. The amplitudes are normalized and plotted with regular spacing.

multiplets ${ }_{0} S_{2}$ and ${ }_{0} S_{3}$ excited by the Chilean earthquake, as observed on a strainmeter (strike 38.4 W of N) at Isabella, California by Benioff et al. (1961). The singlet pair with $m= \pm 1$ has much larger amplitudes than the rest of the ${ }_{0} S_{2}$ multiplet and, similarly, ${ }_{0} S_{3}{ }^{ \pm 2}$ stands out from its multiplet. We also show synthetic relative spectral amplitudes computed for the finite fault and long-period precursor determined by Kanamori and Cipar (1974) from long-period surface waves. The spectral amplitudes do not depend on the precise frequency separation, so for convenience the theoretical amplitudes are plotted with regular spacing. 
The solution for the amplitudes of split modes (Stein and Geller, 1977) is obtained by transforming the spherical harmonic expansion of the excitation from the frame of reference of the source into geographic coordinates. The singlet amplitudes are written so that there are separate factors for source location (latitude and longitude), source depth, fault geometry (strike, dip, and slip direction), receiver location, and the normalized energy of each mode.

For a spheroidal or torsional multiplet and for a step function dislocation with

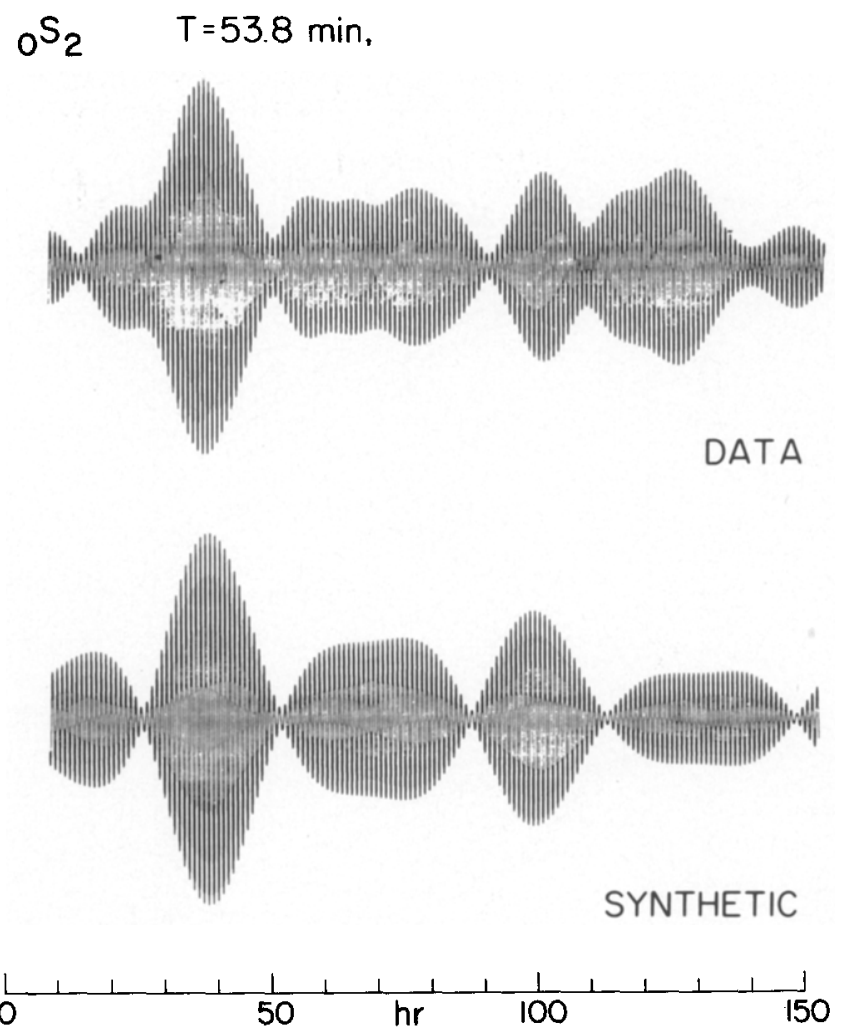

FIG. 2. Data and synthetics for ${ }_{0} \mathrm{~S}_{2}$. The top trace is filtered (tide-removed) data from the first $150 \mathrm{hr}$ of Isabella strain record of the Chilean earthquake. The lower trace is the synthetic seismogram, including the effects of splitting. The synthetic was tapered and filtered in the same way as the data.

unit moment, the displacement or strain component, summing over modes with angular order $l$ and azimuthal order $m$, is given to zeroeth order by

$$
U(r, t)=\sum_{m=-l}^{l}\left[E_{l m}(\vec{r}) e^{i \omega_{l m} t}+E_{l m}^{*}(\vec{r}) e^{-i \omega_{l m} t}\right] e^{-\omega_{l} t / 2 Q_{l}}
$$

where $\omega_{l m}$ is the eigenfrequency of the mode. The overtone number, $n$, will be suppressed for convenience throughout this section. The attenuation factor is assumed to be the same for the entire multiplet. The displacement or strain spectral density of the $l m$ th mode, $E_{l m}$, is given explicity by Stein and Geller (1977). The only perturbations which are important at long periods, rotation and ellipticity, are both symmetric about the rotation axis. To find the displacement, we need the excitation coefficients, which are obtained from the excitation coefficients used for a nonrotating earth in a frame of reference centered on the earthquake source, from 
the results of Saito (1967). We then transform the excitation coefficients from the source coordinates to the geographic coordinates using the rotation matrix elements.

These results make it possible to synthesize the complex time series resulting from the interference of the singlets. Figure 2 shows the observed time series for the ${ }_{0} S_{2}$ multiplet (obtained by narrow band filtering the Isabella record of the 1960 Chilean earthquake) and the synthetic time series computed for the spectral amplitudes shown in Figure 1. The general envelope shape of these modes match the data quite well. Note that the largest peak in the envelope occurs $35 \mathrm{hr}$ after the origin time. The nodal times of the data and synthetics also agree excellently.

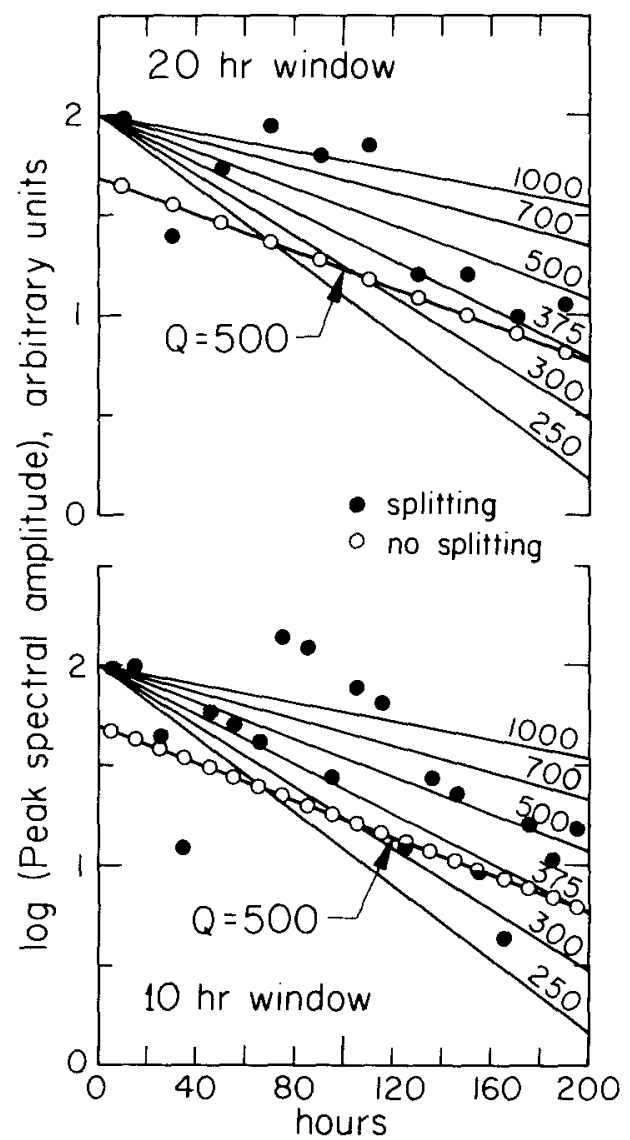

FIG. 3. Results of a numerical test of $Q$ estimation by decay of successive windowed peaks. When there is no splitting (open circles) good results are obtained, but when splitting is present (closed circles) extremely poor and scattered spectral peaks result. The true $Q$ is 500 . Lines for other values are also shown to indicate the errors resulting from treating split modes as unsplit.

Similar figures for other long-period modes are shown by Stein and Geller (1978). This example demonstrates that it is therefore possible to estimate $Q$ despite the interference caused by the splitting, by matching synthetics and data.

\section{Comparison With Frequency Domain $Q$ Determination Methods}

The most common technique for frequency domain $Q$ determination is apparently the use of the decay of successively windowed peak spectral amplitudes. The successive peaks method cannot be used to get reliable $Q$ values when splitting is present. To demonstrate this we will conduct a numerical experiment of the validity 
of the successive peak method in a case where we exactly know the $Q$-namely, displacement or strain synthetics calculated from equation (1). All singlets have the same $Q_{l}$ as long as lateral heterogeneity is not present (Gilbert and Backus, 1965)

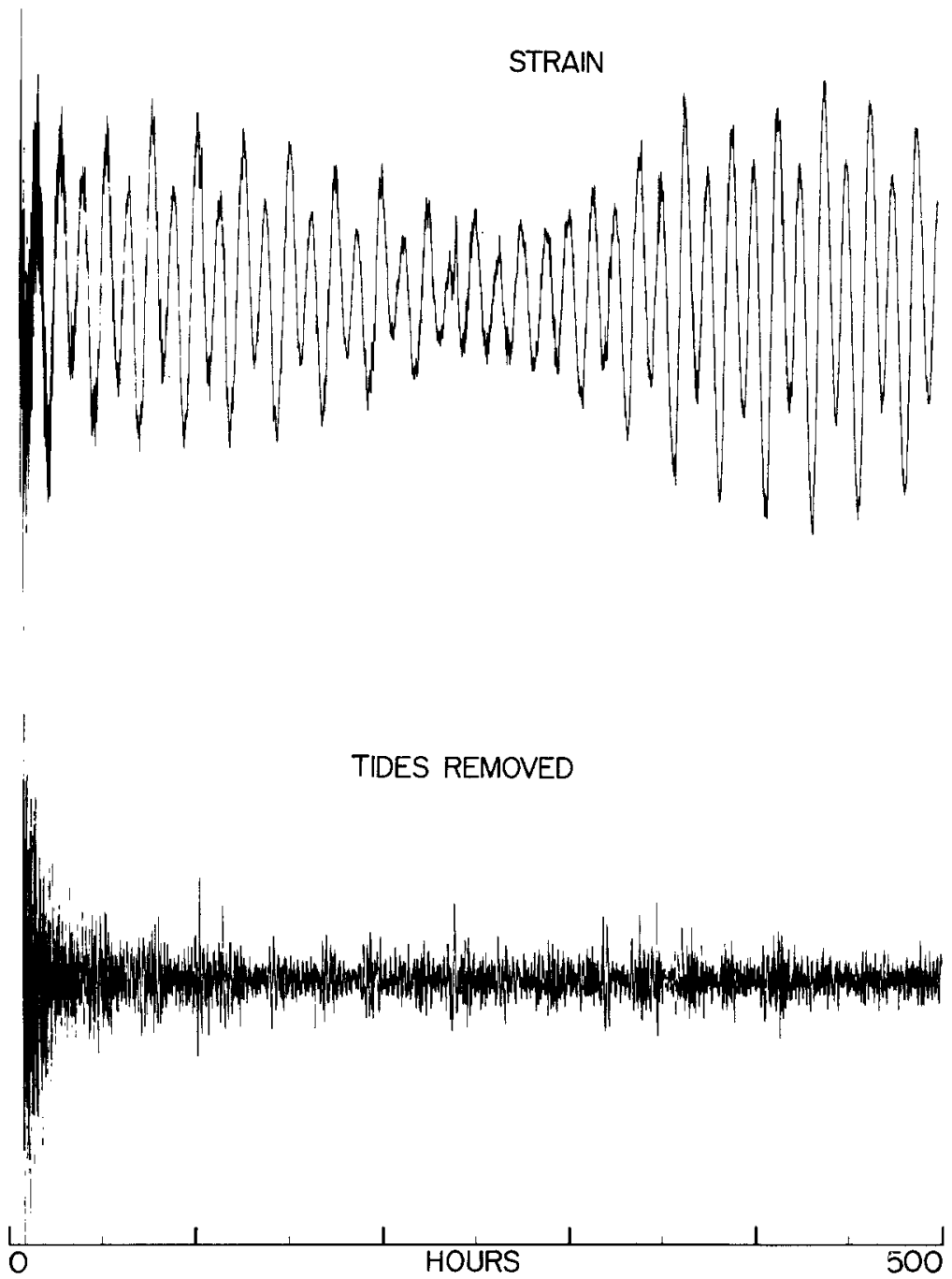

FIG. 4. Isabella strain record of the Chilean earthquake (top trace) and the high-passed record with tides removed (bottom trace). The origin time of this figure, and all others for the Chilean earthquake, is $1911 \mathrm{hr}, 22$ May 1960 , the origin time of the main shock. The digitized record (top) begins $289 \mathrm{~min}$ later, at $0000 \mathrm{hr}, 23 \mathrm{May}$. The high-passed record (bottom) starts another $6 \mathrm{hr}$ later.

and the attenuation factor is included to zeroeth order. For positive $\omega$, the Fourier transform of equation (1) is

$$
U_{l}(\omega)=\left.\sum_{m=-l}^{l} E_{l m} \frac{e^{i \omega_{l m} t-i \omega t-\omega_{l} t / 2 Q}}{i \omega_{l m}-i \omega-\omega_{l} / 2 Q}\right|_{T_{1}} ^{T_{2}}
$$

where $T_{1}$ and $T_{2}$ are the start and end of the windows.

We conduct a numerical experiment for ${ }_{0} S_{3}$, using the modal amplitudes, $E_{3 m}$, for our source model of the Chilean earthquake and a receiver at Isabella. We use the 
preceding equation to obtain the spectrum for any particular time window, and then evaluate the spectral amplitude numerically to find the peak spectral amplitude. In order to guard against the possibility that our result is an artifact of some particular window length, we make tests with two different window lengths, $20 \mathrm{hr}$ and $10 \mathrm{hr}$. Both tests demonstrate that the $Q$ estimates made from the decay of peak spectral amplitude are extremely unreliable and scattered when splitting is a significant effect.

The results of the numerical experiment are shown in Figure 3. For all cases, $Q$ $=500$ is used. The top plot shows the test with a $20-\mathrm{hr}$ window. The peak spectral amplitude for each 20-hr interval (e.g., 0 to $20 \mathrm{hr}, 20$ to 40 , etc.) is plotted at the
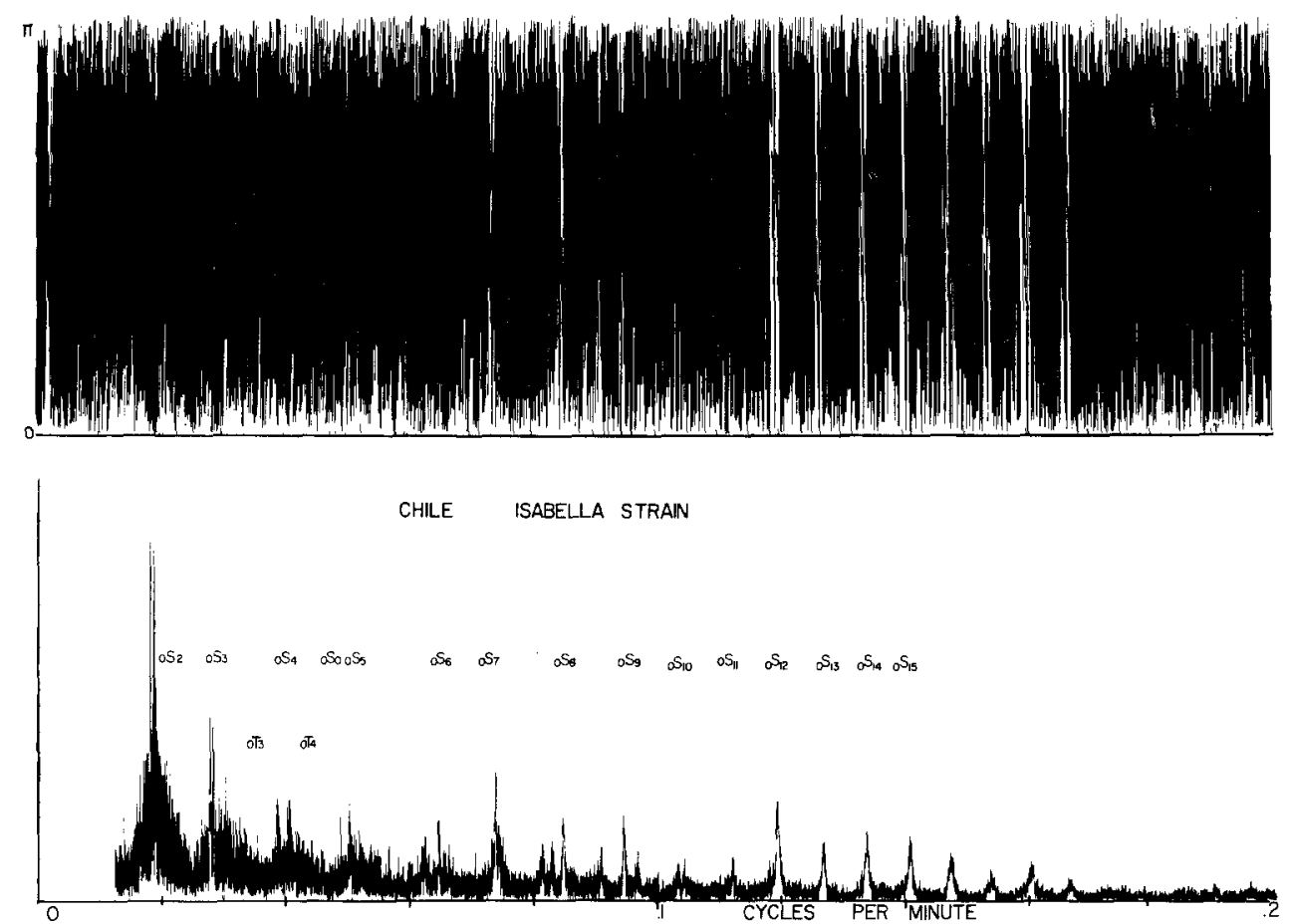

FIG. 5. Amplitude and phase spectra of the Isabella strain record. Frequencies are in cycles per minute. Note the $\pi$ phase change across the resonance peak.

center of the interval. Two cases were considered. When there is no rotational splitting (all the singlets have the same eigenfrequency, $\omega_{l m}=\omega_{l}$ ), the peak amplitudes (the open circles) are well behaved, falling exactly on the line for $Q=500$. On the other hand, the peak amplitudes for the case of split modes (closed circles) are very badly scattered and do not even decay monotonically. Even though the peak spectral amplitudes are exactly calculated, with no noise, and the $Q$ is set exactly to 500 it is completely.impossible to recover the $Q$ from the peak amplitudes. The same is true of the 10-hr window (bottom of Figure 3). Again, although the peak spectral amplitudes for the unsplit modes lie exactly on the $Q=500$ curve, in the split case the peak spectral amplitudes are very badly scattered and unusable for a reliable $Q$ determination. Curves for other values of $Q$ demonstrate the range of possible error. 


\section{DATA}

The two data sets most suitable for measuring the attenuation of the longest period modes are the Isabella strain meter record of the 1960 Chilean earthquake, and the UCLA gravity meter record of the 1964 Alaskan earthquake. Although neither instrument was ideal for this purpose, the two earthquakes were large

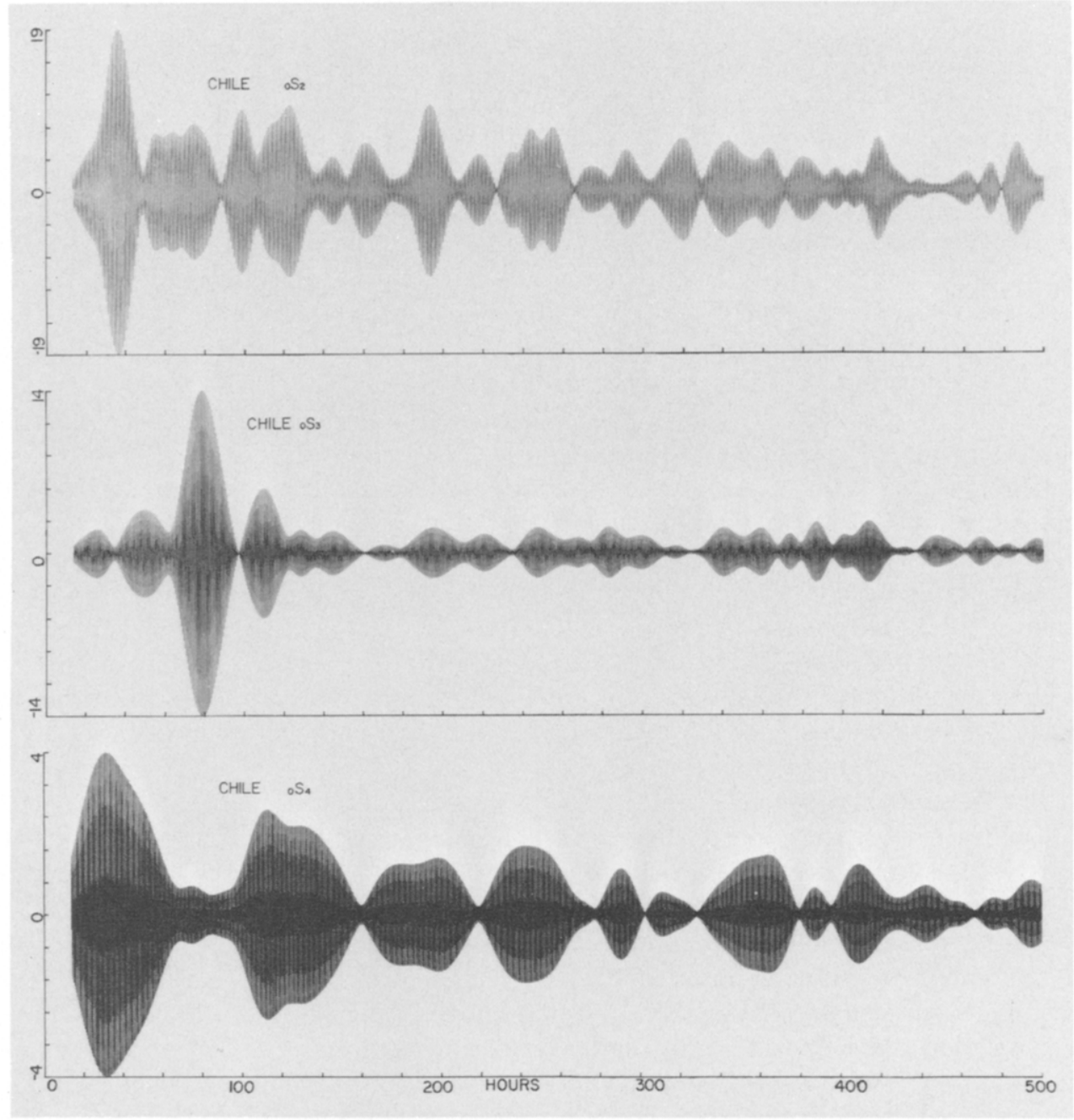

Fig. 6. Band-pass filtered data from the Isabella record for ${ }_{0} S_{2},{ }_{0} S_{3}$, and ${ }_{0} S_{4}$. The amplitude is in digital units.

enough to generate acceptable records for long enough time periods (several hundred hours). Even so, the signal-to-noise ratios for the low-order modes are low, and limit the possible data analysis substantially. Here, then, techniques are demonstrated which will be far more effective when data from the new long-period IDA network become available for large earthquakes.

The Caltech strainmeter at Isabella, California (Benioff, 1959, 1960) is the longest ultra long-period record of the Chilean earthquake. This record was used by Benioff et al. (1961) and Smith (1961) in their classic studies which yielded the first spectra 
showing the longest period modes. Figure 4 shows $500 \mathrm{hr}$ of the strain record, which was filtered electronically prior to recording to reduce the tidal amplitudes. The record (top) was processed to remove tides by twice subtracting 3-hr running averages. The resulting record (bottom) was then tapered at both ends (with a cosine window over the first and last 2 per cent of the data) for spectral analysis.

The amplitude and phase spectra for the entire record in Figure 4 are shown in Figure 5. Frequencies are given in cycles per minute, and some of the modes are labeled. Modes appear as peaks in the amplitude spectra, and as "white" areas in the phase spectrum. (This is because of the $\pi$ phase shift across a resonance peak. The phase is incoherent except near a well developed peak, yielding the dark areas on the plot, and coherent across a well developed peak.) This can be seen best for "clean" peaks, for example ${ }_{0} S_{12}(0.12 \mathrm{cpm})$. Noisier and more jagged peaks, for example ${ }_{0} S_{2}$, do not stand out as well in the phase spectrum. (Each singlet should ideally have its own resonance curve; they will interfere unless the singlets are well separated.) The quality of the longest period peaks proved a limiting factor in the later analysis. Some modes are barely resolvable on this record, but can be resolved on the UCLA gravity record of the Alaskan earthquake. Torsional modes are generally indistinct on the Isabella record.

The tapered spectrum was filtered to isolate individual multiplets, as shown in the following set of figures (6 and 7). The filter windows used in the analysis are listed in Table 1. The zero phase shift filter fell off to $e^{-1}$ at either end of the passband, and to zero outside the passband. The filtered data show the characteristic beating patterns resulting from singlet interference.

The vertical scale on each figure gives the trace amplitude in digital units. The one unit level can usually be regarded as a nominal noise level, and is definitely a lower bound. As discussed later, in some cases the true noise level appears to be higher. Some modes remain above the noise level for extended periods of time. In contrast, some modes, such as ${ }_{0} T_{3}$ and ${ }_{0} T_{4}$ (Figure 7) are barely above the noise level initially and soon decay below it.

The second data set used here is the UCLA gravity meter record of the 1964 Alaskan earthquake (Slichter, 1967). Two such meters were operating in 1964, one of which (meter 7) yielded data far inferior to the other (meter 4). Only the meter 4 record is used in the later analysis.

The raw record (Figure 8) contains a number of large amplitude glitches which have periods of tens of minutes. Because this could significantly bias the results for the long-period modes, these glitches were removed. Wiggins and Miller (1972) used a prediction error operator to isolate and remove glitches. We fit a straight line across the affected areas (Figure 7, bottom), which also yielded excellent results. Several small single point glitches were not removed, but these have only a negligible effect on the spectrum. The amplitude and phase spectra of the entire deglitched record are given in Figure 9. Filtered time series and envelopes (Figures 10 and 11) were obtained for the four spheroidal modes, using the same filter windows as for the Chile data.

\section{ANALYSIS}

To analyze this data, synthetic seismograms for each multiplet were generated using the method of Stein and Geller (1977). These were then compared to the data to provide an estimate of $Q$. The synthetics were calculated for the appropriate source mechanism, without including the effects of attenuation (particularly for the low-order modes, the synthetics are very insensitive to changes in the source model, Geller and Stein, 1977). A range of different $Q$ values were then applied to the " $Q$ - 


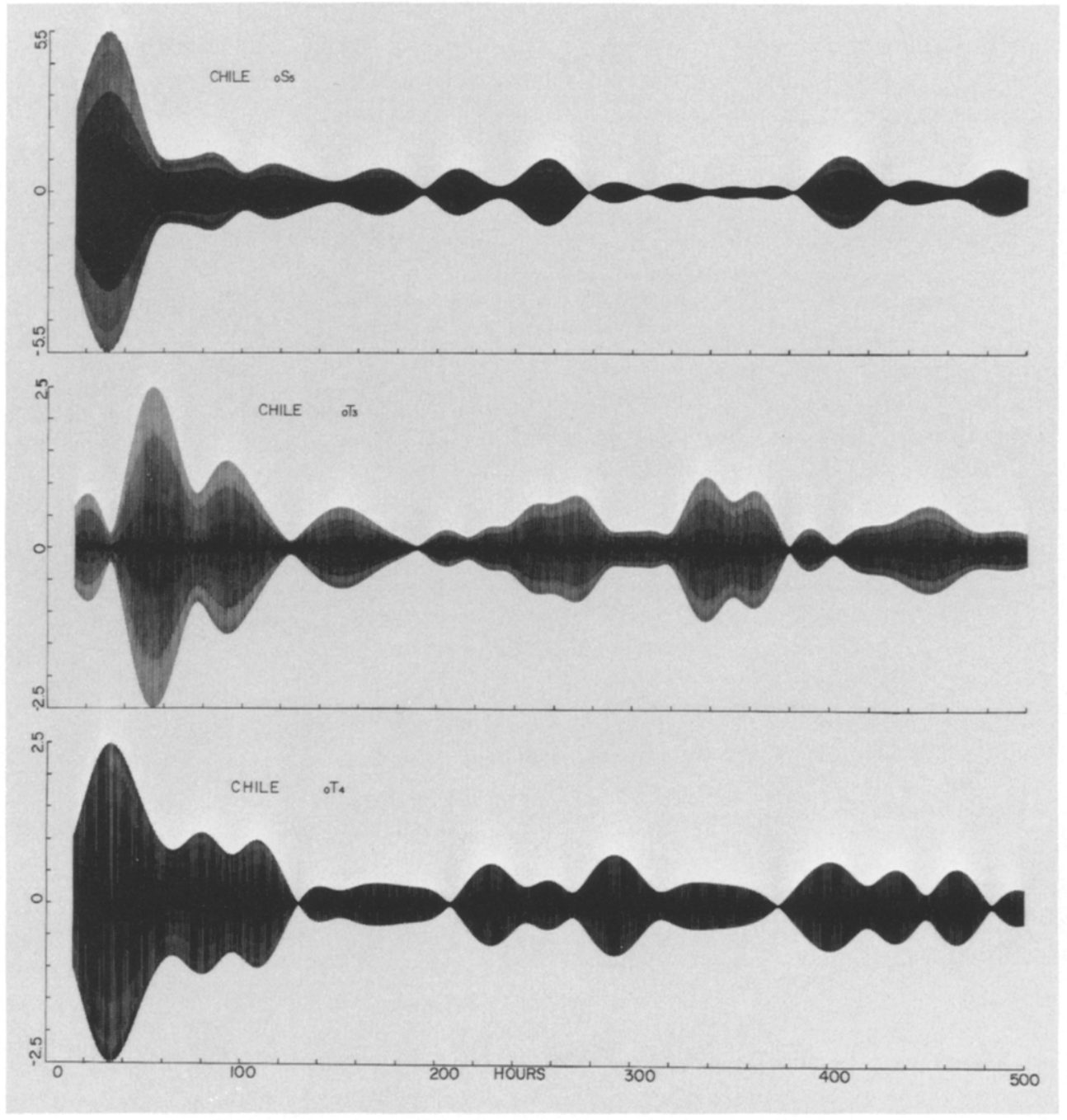

Fig. 7. Band-pass filtered data from the Isabella record for ${ }_{0} S_{5},{ }_{0} T_{3}$, and ${ }_{0} T_{4}$. The amplitude is in digital units.

TABLE 1

\begin{tabular}{ccc}
\multicolumn{3}{c}{ Filter PassBands } \\
\hline Mode & $\begin{array}{c}\text { Min. Frequency } \\
\text { (cpm) }\end{array}$ & $\begin{array}{c}\text { Max. Frequency } \\
\text { (cpm) }\end{array}$ \\
\hline${ }_{0} S_{2}$ & 0.01750 & 0.01950 \\
${ }_{0} S_{3}$ & 0.02726 & 0.02882 \\
${ }_{0} S_{4}$ & 0.03821 & 0.03935 \\
${ }_{0} S_{5}$ & 0.04986 & 0.05070 \\
${ }_{0} T_{3}$ & 0.03481 & 0.03566 \\
${ }_{0} T_{4}$ & 0.04565 & 0.04640 \\
\hline
\end{tabular}

less" synthetics, and the resulting time series were then tapered and filtered in the same way as the data.

The eigenfrequency of each singlet is computed using Anderson and Hart's (1976) values for the unperturbed eigenfrequencies and Dahlen's (1968) rotational splitting parameters. The elliptical splitting parameters are not used, due to the difficulties involved in previous calculations of these parameters (Woodhouse, 1976). Dahlen 
(personal communication) states that more accurate recent computations show that the spheroidal mode elliptical splitting parameters may be neglected for our purposes. Accurate values have not yet been published for the elliptical splitting of torsional modes, or for splitting due to lateral heterogeneities.

The Hilbert transform envelopes of both data and synthetics were smoothed with running averages, for ease of comparison. These smoothed envelopes have the
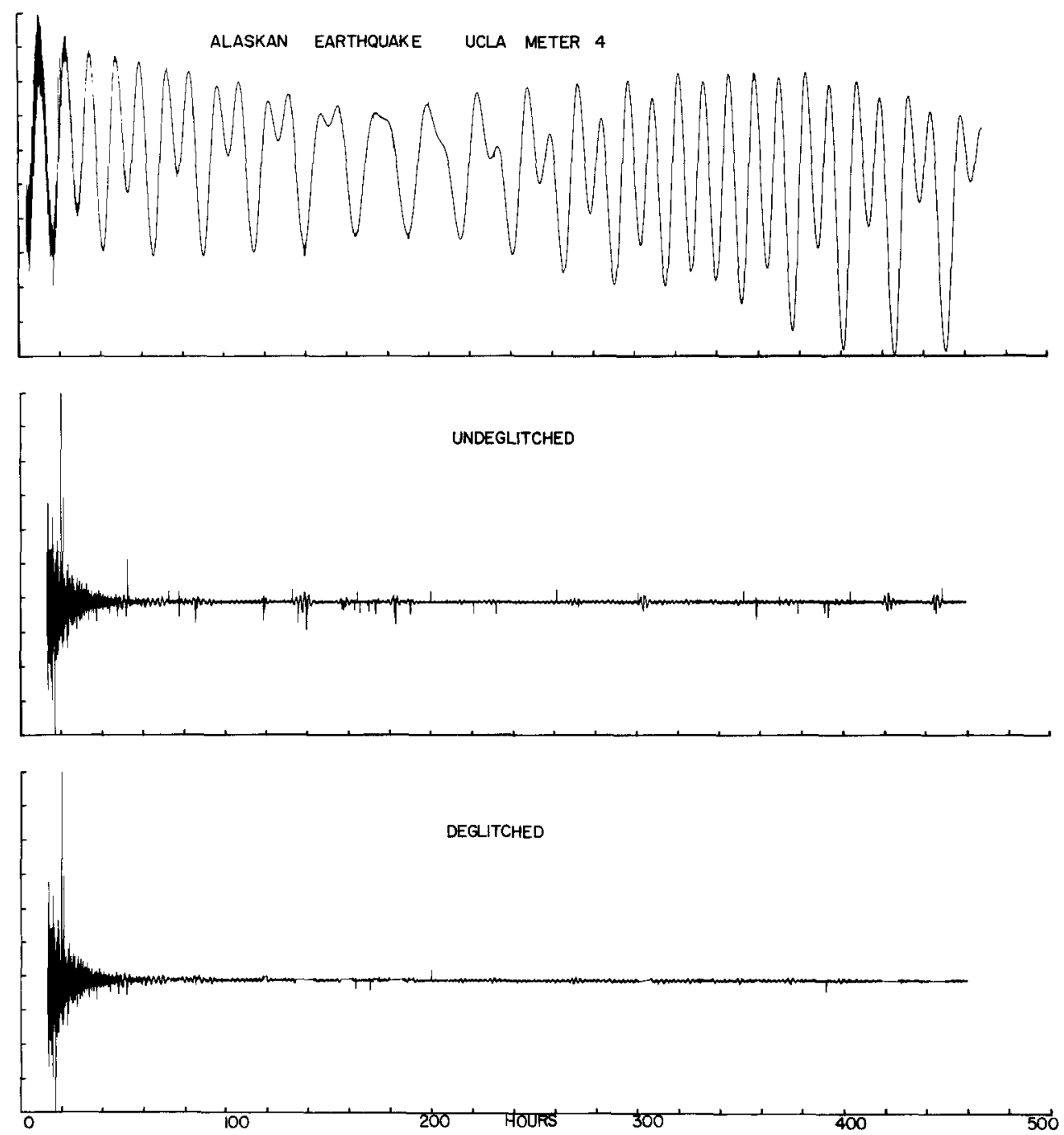

FIG. 8. UCLA gravity (meter 4) record of the Alaskan earthquake (top). The origin time of this figure, and all other Alaska figures, is $0336 \mathrm{hr}, 28$ March 1964, the origin time of the main shock. The digitized record begins $256 \mathrm{~min}$ later. The middle trace shows the large glitches present after tide removal; the lower trace shows the final deglitched record.

information needed for studying the beat patterns and their time decay, and are much easier to store and manipulate than the entire time series. In addition, due to noise and lateral heterogeneity, as time progresses the general decay of data and synthetics are similar but individual beats are sometimes not well in phase.

For the Chilean synthetics, we use the source mechanism determined by Kanamori and Cipar (1974) from long-period surface waves. The rupture was initiated at $38^{\circ} \mathrm{S}$, $286.5^{\circ} \mathrm{E}$ and propagated at $3.5 \mathrm{~km} / \mathrm{sec}$ to $46^{\circ} \mathrm{S}, 286.5^{\circ} \mathrm{E}$, on a fault plane dipping $10^{\circ}$ 

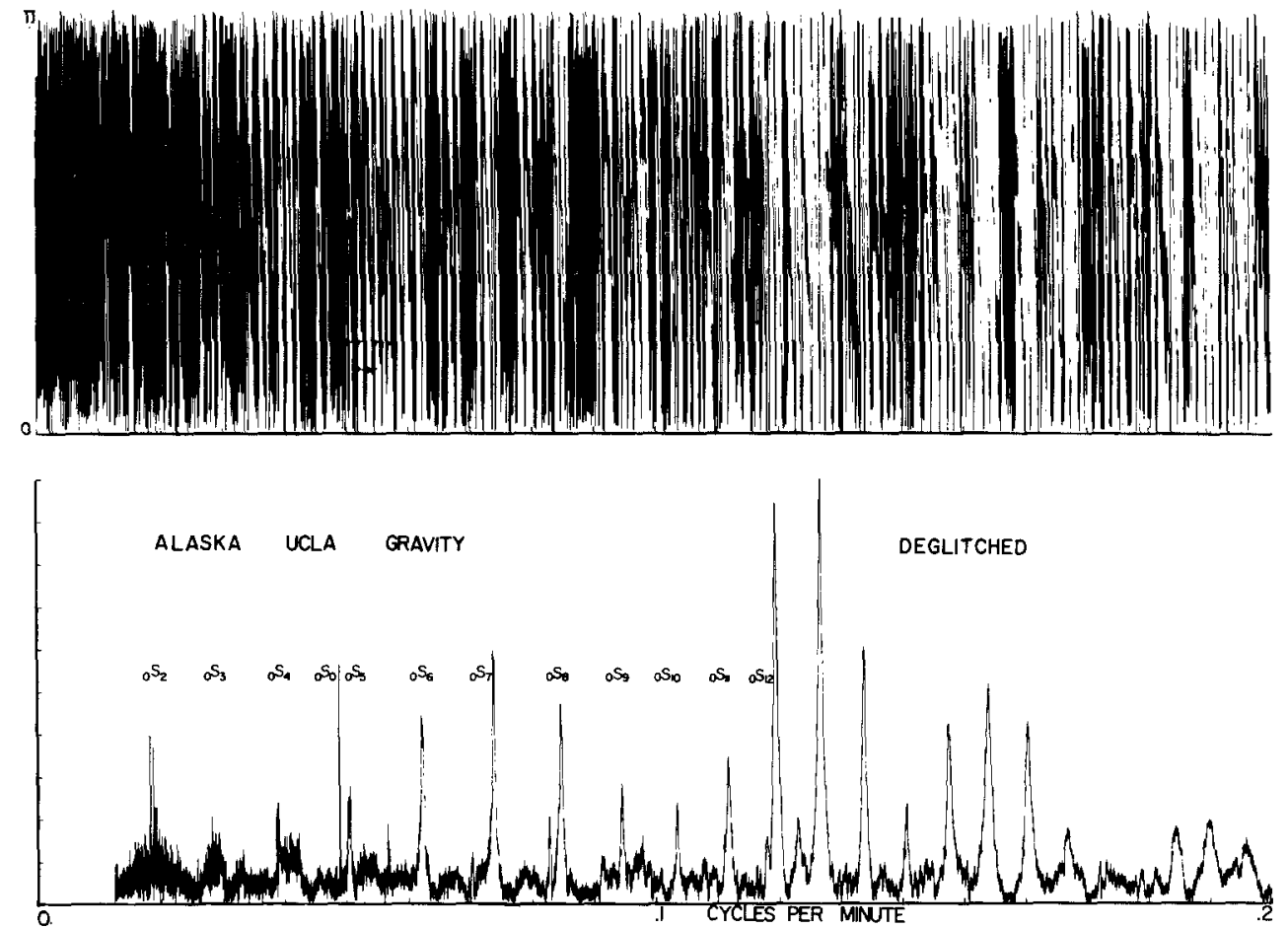

FIG. 9. Amplitude and phase spectra of the UCLA gravity meter record after deglitching. The noise level is reduced substantially. Note the $\pi$ phase shift across the resonance peak.

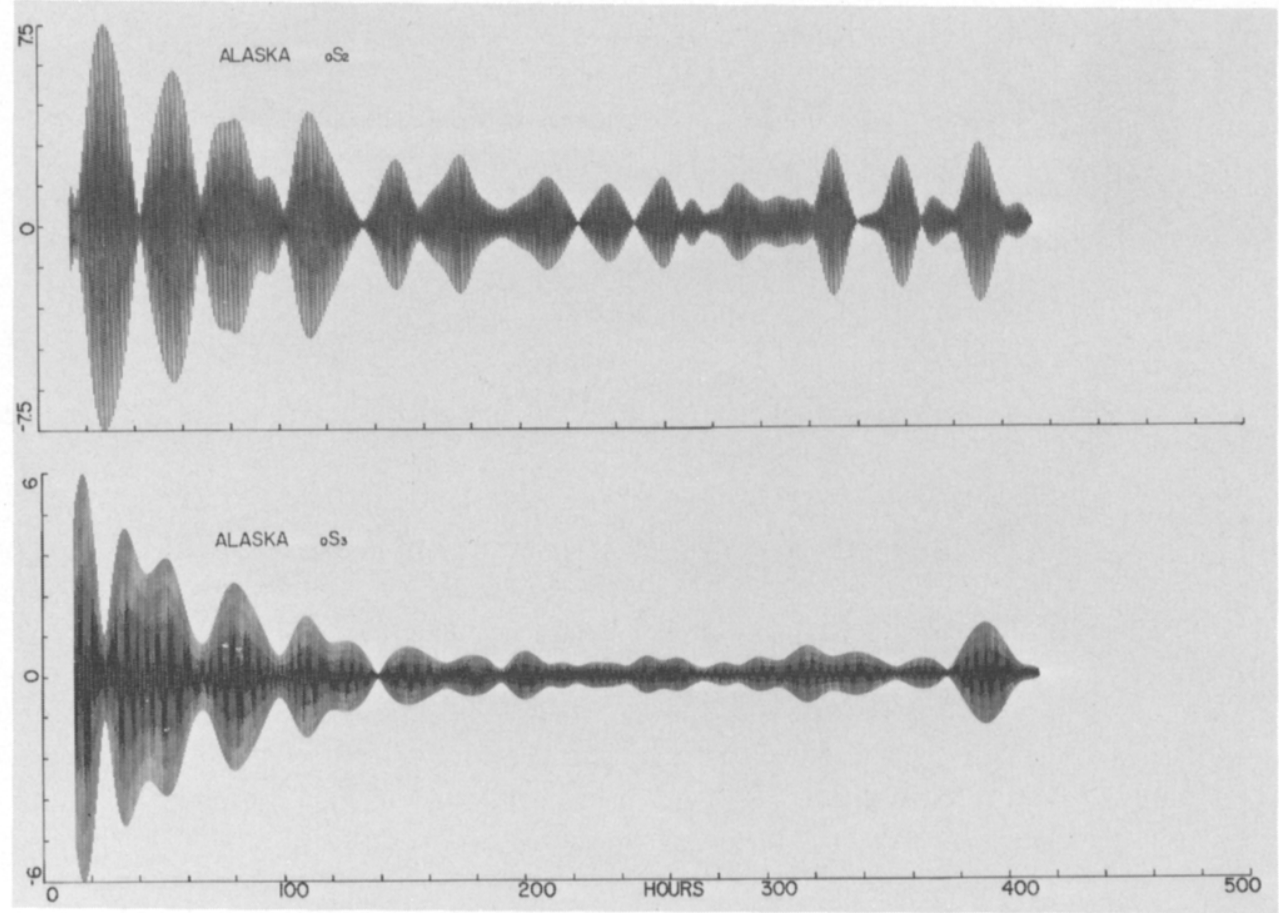

FIG. 10. Band-pass filtered data from the UCLA gravity record for ${ }_{0} S_{2}$ and ${ }_{0} S_{3}$. The amplitude is in digital units. 
east and striking $\mathrm{N} 10^{\circ} \mathrm{E}$. (We approximate the finite source by five point sources at a depth of $55 \mathrm{~km}$.) The slip angle is $90^{\circ}$, a pure thrust motion. We are also including a precursory slip (Kanamori and Cipar, 1974; Kanamori and Anderson, 1975) at $41.5^{\circ} \mathrm{S}, 285.7^{\circ} \mathrm{E}$ with a rise time of 5 min starting $15 \mathrm{~min}$ before the main shock, and with a moment equal to that of the main shock.

For the Alaskan synthetics, the fault parameters used are those $\left(\rho=114^{\circ}, \lambda=\right.$ $90^{\circ}, \delta=20^{\circ}, \theta_{s}=29.9^{\circ}, \phi_{s}=212.4^{\circ}, L=500 \mathrm{~km}, V_{R}=3.5 \mathrm{~km} / \mathrm{sec}$ ) determined by Kanamori (1970). The finite source is approximated in the same way as for Chile.

All the figures show the smoothed Hilbert transforms of the data and synthetics, for six values of $Q: 300,400,500,600,750,1000$. Synthetics with $Q$ 's of 1250 and 1500 were added for ${ }_{0} S_{2}$. The Chilean results for ${ }_{0} S_{2-0} S_{5},{ }_{0} T_{3}$, and ${ }_{0} T_{4}$ are presented first (Figures 12 to 14).

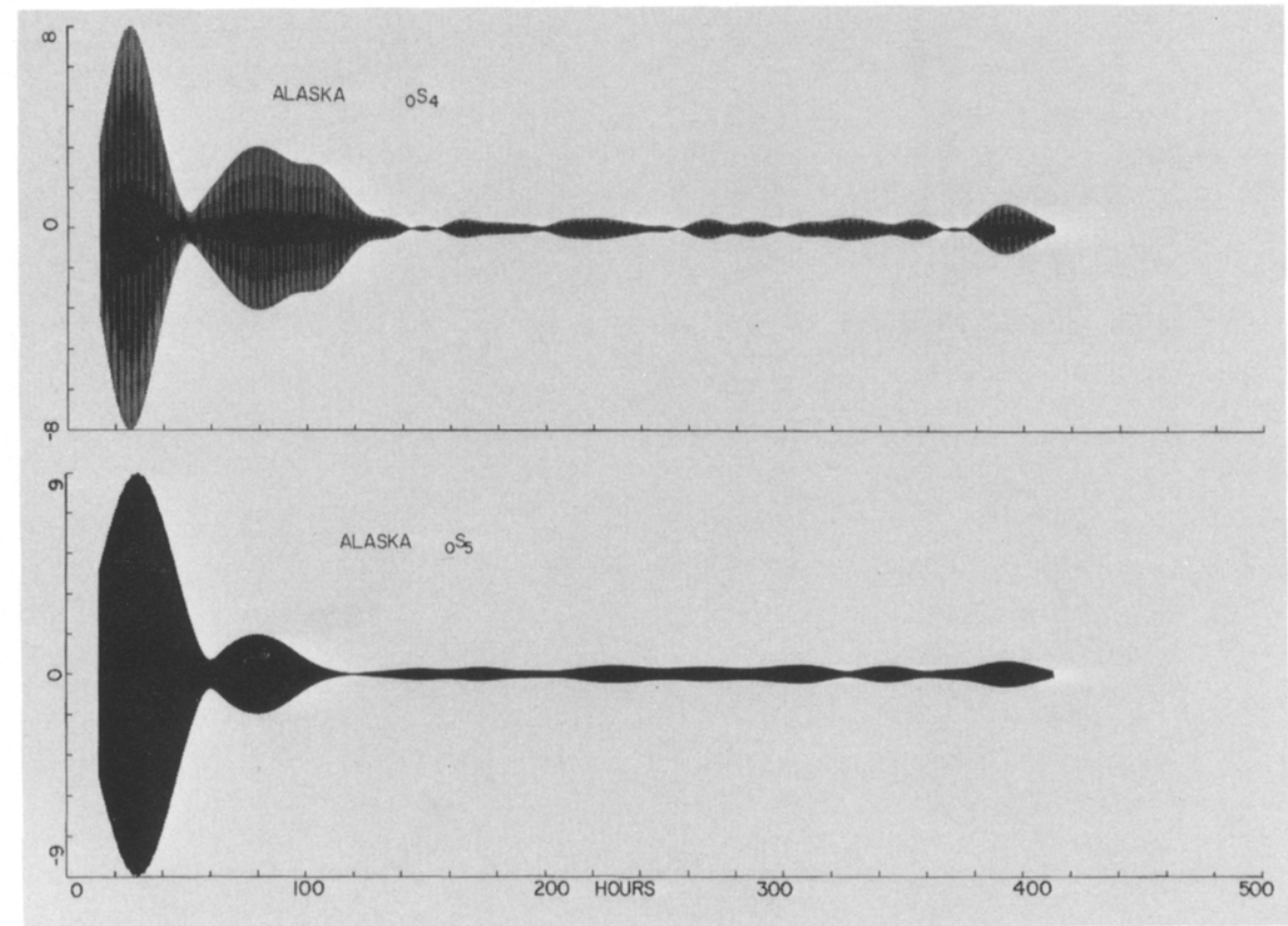

FIG. 11. Band-pass filtered data from the UCLA gravity meter record for ${ }_{0} S_{4}$ and ${ }_{0} S_{5}$. Time is in hours, and amplitude is in digital units.

The noise levels marked on each plot are assigned somewhat subjectively. The highest noise level, four digital units for ${ }_{0} S_{2}$, seems appropriate in that the signal amplitude stabilizes at about this level. A major factor in this was a comparison to ${ }_{0} S_{2}$ for the UCLA Alaska record. This is consistent with the fact that the noise level for the ${ }_{0} S_{2}$ multiplet (see the spectrum, Figure 4) seems higher than for the other modes. For the other Isabella records, the noise level is assumed to be the nominal one digital unit noise level. The smoothing intervals were also chosen empirically, to smooth out major irregularities and noise bursts in the data.

$Q$ is measured by comparing the smoothed data to the smoothed synthetic curves, which bracket the data. The best-fitting $Q$ is that obtained by using the maximum record length before the noise level is reached. Our $Q$ values are tabulated in Table 2 along with those obtained by previous investigations. 


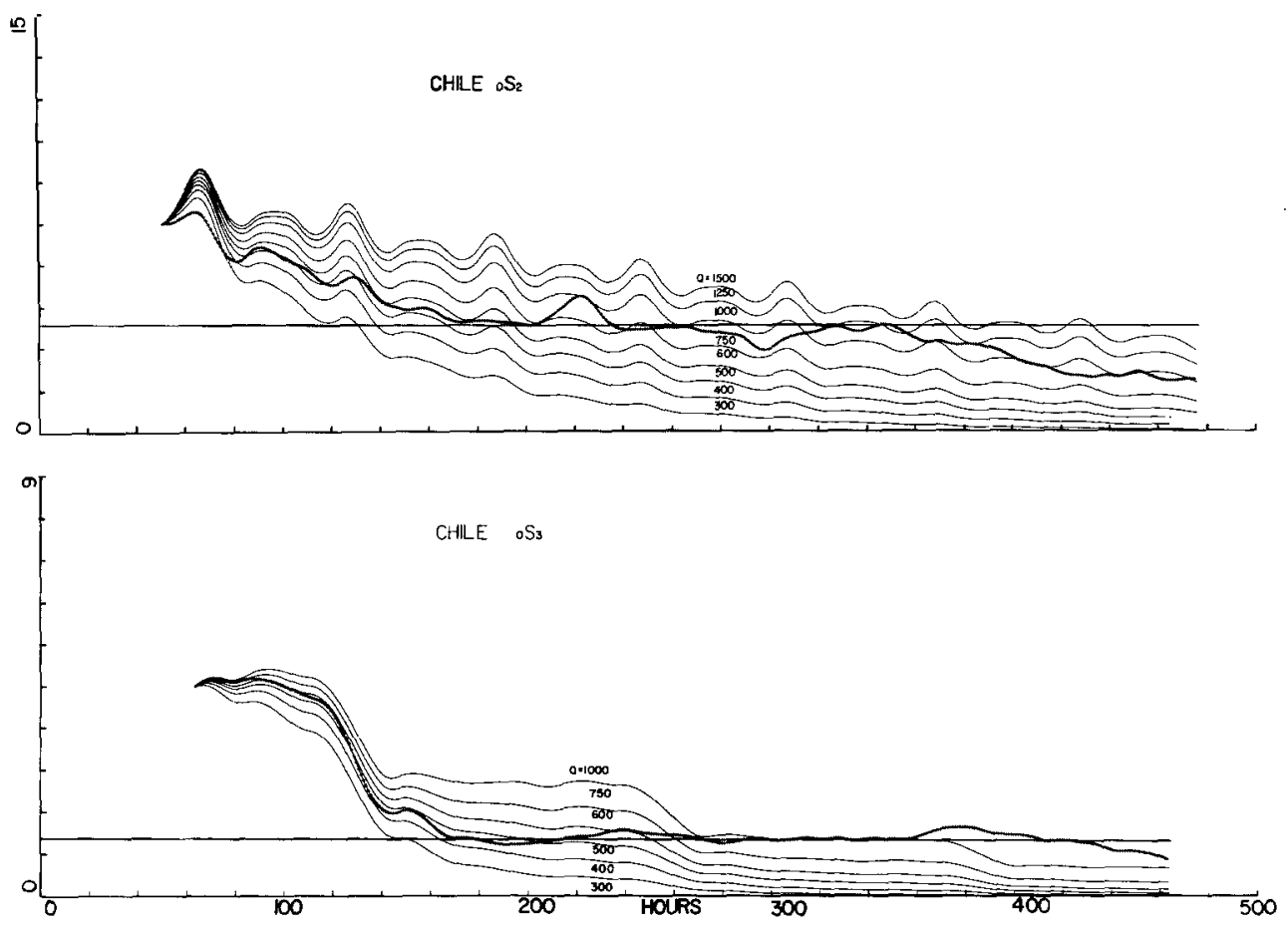

FIG. 12. (Top) Smoothed data and synthetic envelopes for the Isabella strain record of the Chilean earthquake. All envelopes are smoothed with a 75-hr running average. Synthetics were calculated for 8 different values of $Q$. The noise level is 4 digital units. The time scale is the same as in Figure 4. (Bottom) Data and synthetics for ${ }_{0} S_{3}$. The noise level is one digital unit, and the smoothing window is $100 \mathrm{hr}$ long.
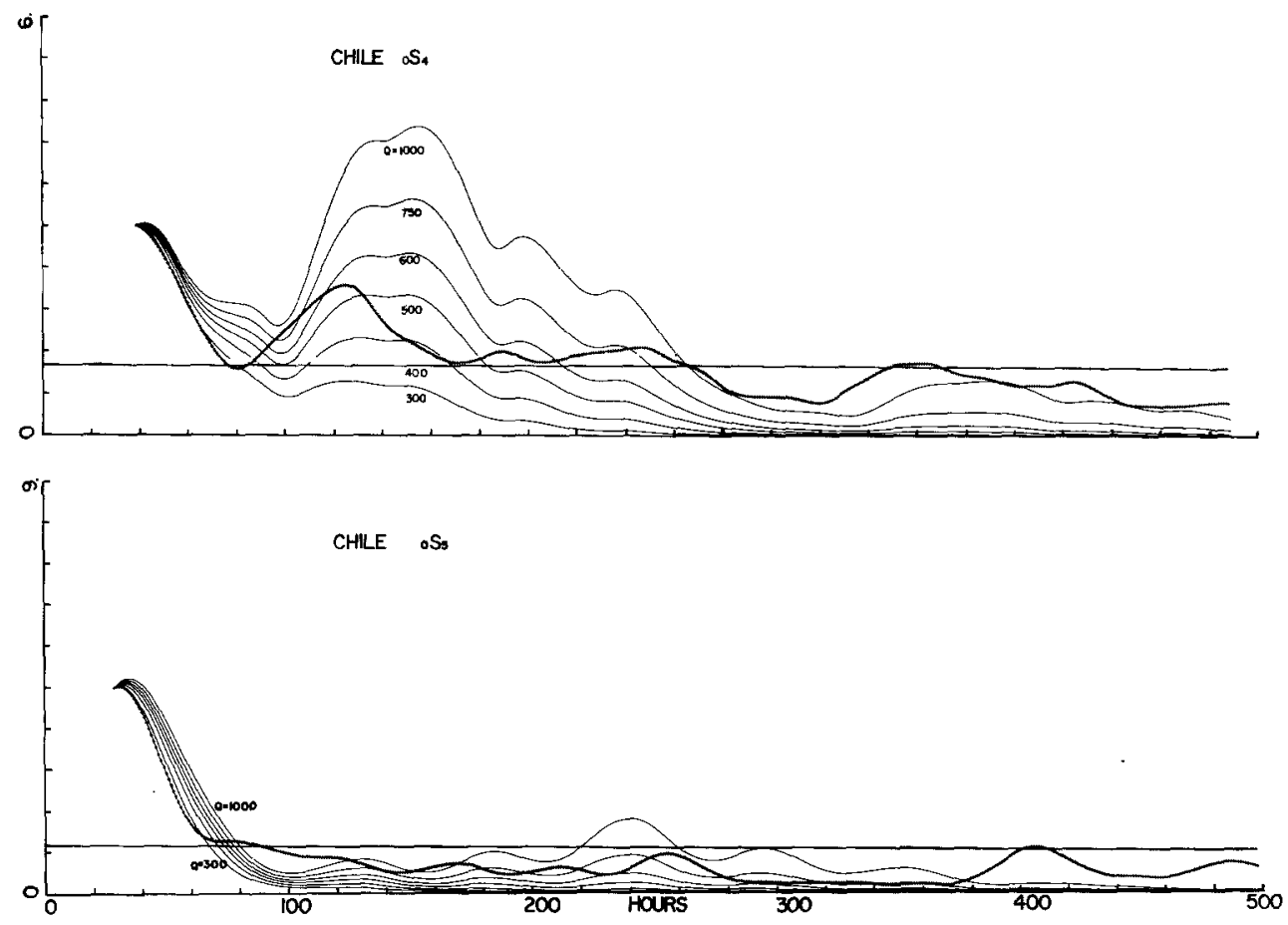

FIG. 13. (Top) Data and synthetics for ${ }_{0} S_{4}$. The noise level is one digital unit, and the smoothing window is $50 \mathrm{hr}$ long. (Bottom) Data and synthetics for ${ }_{0} S_{5}$. The noise level is one digital unit, and the smoothing window is $30 \mathrm{hr}$ long. $Q$ values are $1000,750,600,500,400$, and 300 . 
This procedure works well for modes with adequate signal levels, in this case the four Chile spheroidal mode records. The results are much less satisfactory for the torsional modes (Figure 4), but these data are included for completeness. This is hardly surprising as the torsional mode peaks in the spectrum (Figure 3) are almost undetectable.

Figures 15 and 16 show the four corresponding spheroidal modes from the UCLA gravity record for the Alaskan earthquake. In general, the signal level is adequate and the noise level is considered to be one digital unit. Our $Q$ values are also listed in Table 2.
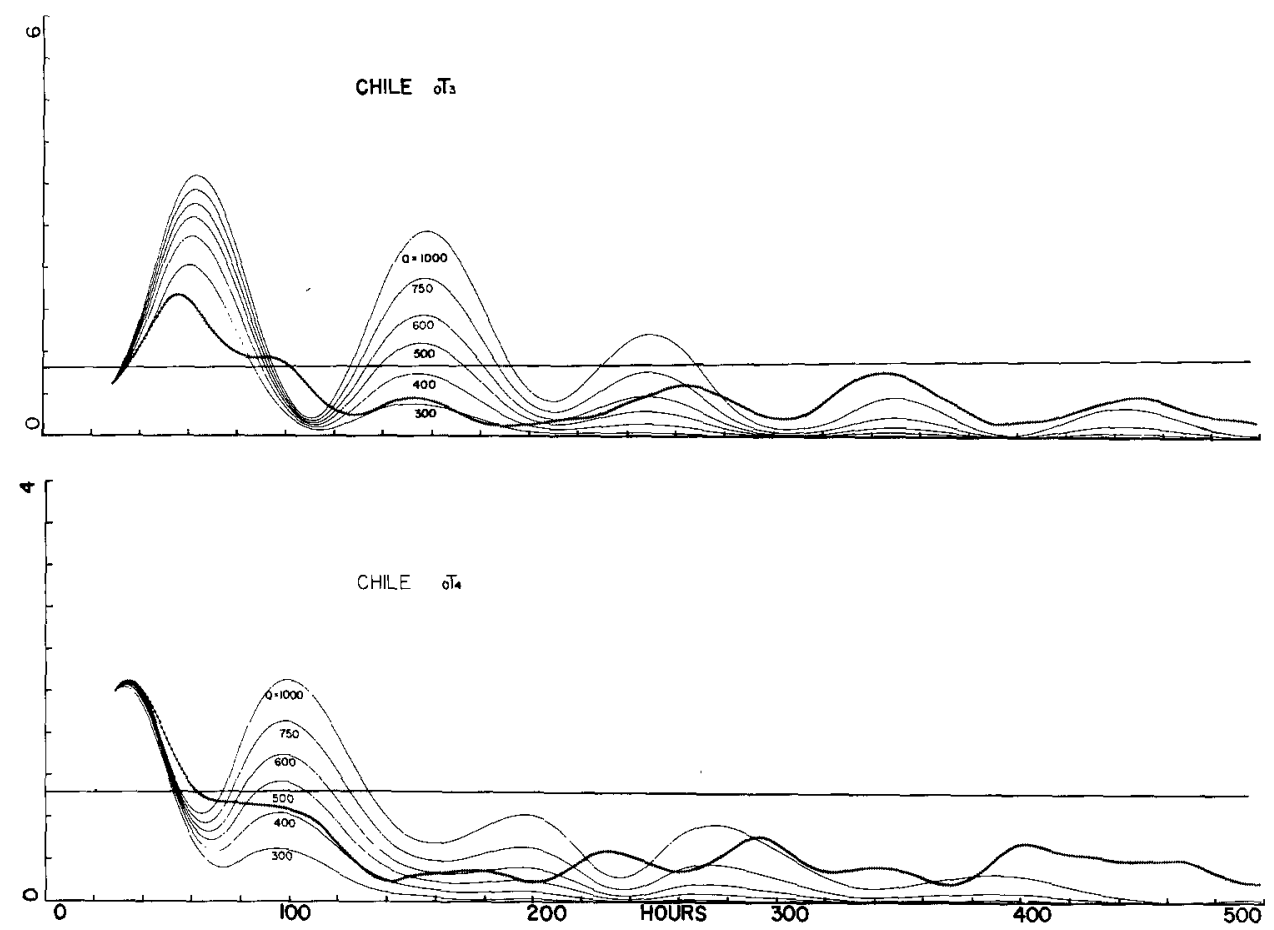

FIG. 14. (Top) Data and synthetics for ${ }_{0} T_{3}$. The noise level is one digital unit, and the smoothing window is $30 \mathrm{hr}$ long. (Bottom) Data and synthetics for ${ }_{0} T_{4}$. The noise level is one digital unit, and the smoothing window is $30 \mathrm{hr}$ long.

\section{Discussion}

$Q$ values estimated from the data in the previous section are tabulated in Table 2. These were obtained from the portions of the record above the noise level. The spheroidal modes have error bounds of approximately \pm 100 , and the torsional modes have error bounds of approximately \pm 200 .

Table 2 also lists the results obtained by previous studies. In general, the results of this study do not differ substantially from the other values. The major difference is for the $Q$ of ${ }_{0} S_{2}$, which has substantial significance for gross earth models.

Buland and Gilbert (1978) argued against the existence of a low $Q$ zone at the base of the mantle, from the high value (775 to 815) of the $Q$ of $S_{2}$. Our results (425 to 550) are in general accord with Slichter's (1967) and Sailor and Dziewonski's (1978), and are much lower than 800 . Such data may be consistent with the presence of a low $Q$ zone.

The scatter in $Q$ values reported by various investigators is clearly due to the limited quality of the relatively small body of data available for such ultra longperiod studies. Any $Q$ measurement technique will thus face substantial difficulties. 

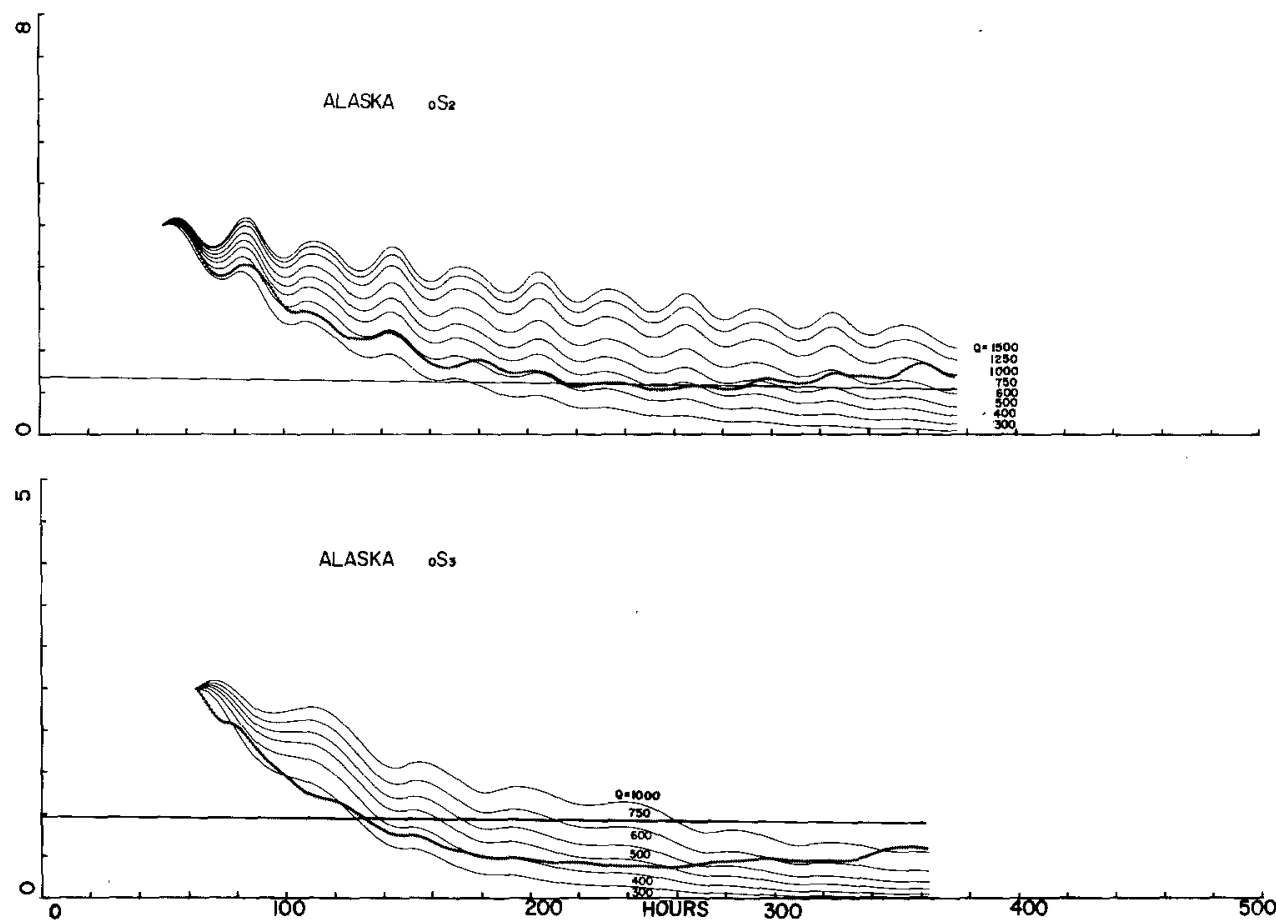

Frg. 15. (Top) Data and synthetics for $S_{2}$ on the UCLA gravity record of the Alaskan earthquake Time is in hours since the origin time $(0336 \mathrm{hr}, 28 \mathrm{March} \mathrm{1964)}$ on the next five figures. The noise level is one unit, and the smoothing window is $75 \mathrm{hr}$ long. (Bottom) Data and synthetics for o $S_{3}$. The noise level is one unit, and the smoothing window is $100 \mathrm{hr}$.
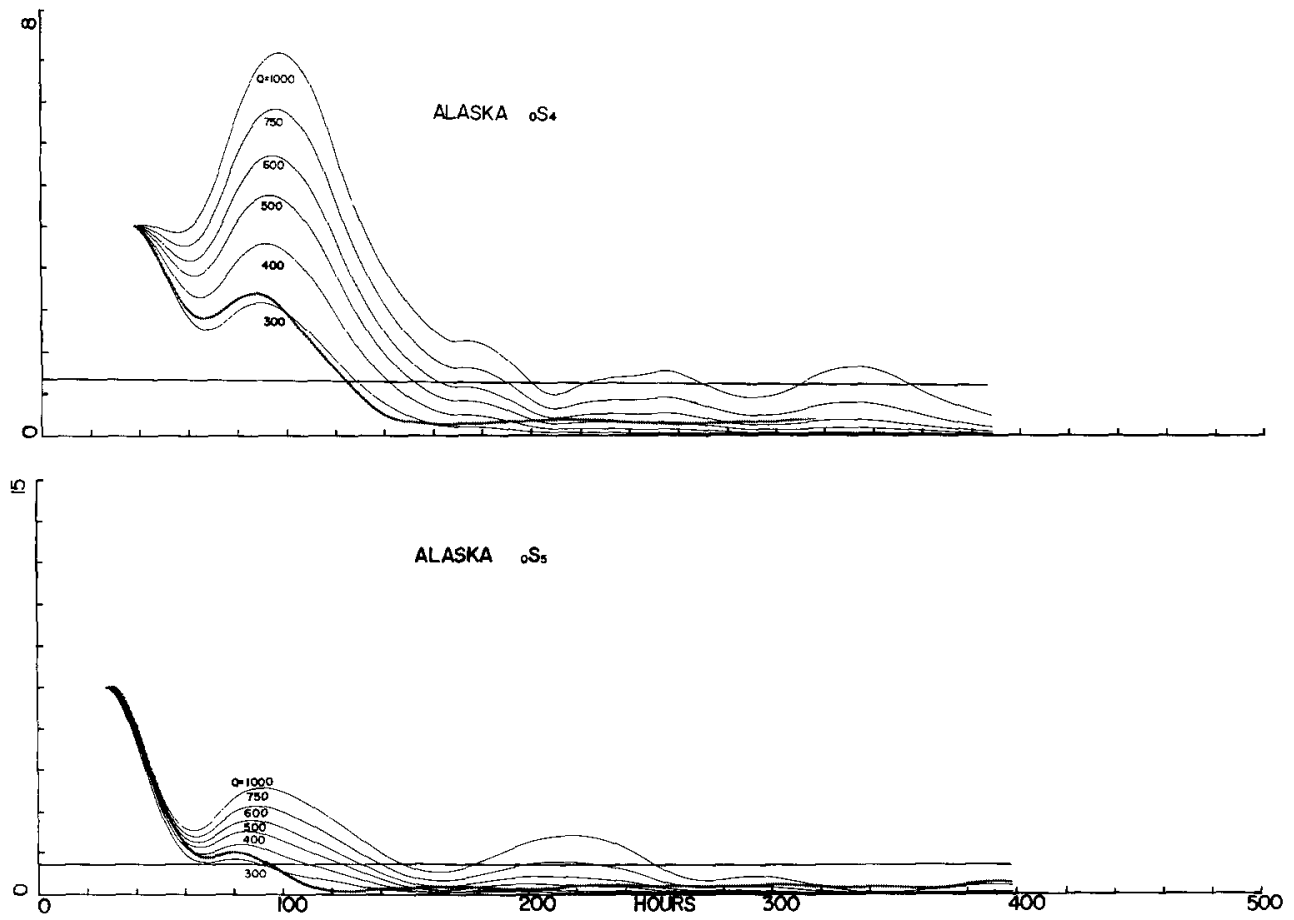

FIg. 16. (Top) Data and synthetics for ${ }_{0} S_{4}$. The noise level is one unit and the smoothing window is $50 \mathrm{hr}$. (Bottom) Data and synthetics for ${ }_{0} S_{5}$. The noise level is one unit and the smoothing window is 30 hr. 
Although our choice of noise level (Figure 12) probably minimizes this problem, the essential limitation is still the signal quality.

Comparison of narrow-band filtered data and synthetic seismograms generated using theoretical results for the excitation of split modes is a valuable technique for $Q$ estimation. Such methods are necessary when it is not possible to isolate individual singlets, and the combined beat pattern must be used. The limitations on this method resulting from the quality of the data from the early 1960's can be removed once high-quality long-period data for large earthquakes becomes available.

TABLE 2

$Q$ VALUES FROM VARIOUS STUdIES

\begin{tabular}{ll}
\hline$S_{2}$ & \\
425 & This study, Alaska/UCLA \\
550 & This study, Chile/Isabella \\
775 & Buland and Gilbert (1978), Alaska/UCLA \\
815 & Buland and Gilbert (1978), Alaska/UCLA \\
556 & Sailor and Dziewonski (1978), Alaska/UCLA \\
500 & Slichter (1967) \\
420 & Smith (1972), Alaska and Chile/Isabella \\
370 & Alsop et al. (1961b), Chile/Ogdensburg \\
$0 S_{3}$ & \\
450 & This study, Chile/Isabella \\
325 & This study, Alaska/UCLA \\
460 & Sailor and Dziewonski (1978), Alaska/UCLA \\
500 & Slichter (1967), UCLA/Alaska \\
450 & Smith (1972), Alaska and Chile/Isabella \\
380 & Benioff et al. (1961), Chile/Isabella \\
$0 S_{4}$ & \\
275 & This study, Alaska/UCLA \\
400 & This study, Chile/Isabella \\
411 & Sailor and Dziewonski (1978), Alaska/UCLA \\
400 & Slichter (1967), Alaska/UCLA \\
330 & Smith (1972), Alaska and Chile/Isabella \\
$0 S_{5}$ & \\
325 & This study, Alaska/UCLA \\
300 & This study, Chile/Isabella \\
352 & Sailor and Dziewonski (1978), Alaska/UCLA \\
300 & Smith (1972), Alaska and Chile/Isabella \\
$0 T_{3}$ & \\
325 & This study, Chile/Isabella \\
370 & Smith (1972), Alaska and Chile/Isabella \\
400 & Smith (1961), Chile/Isabella \\
$0 T_{4}$ & \\
425 & This study, Chile/Isabella \\
290 & Smith (1972), Alaska and Chile/Isabella \\
\hline & \\
\hline 50
\end{tabular}

\section{ACKNOWLEDGMENTS}

We thank Yoshio Fukao, Hiroo Kanamori, Stewart Smith, and Ralph Wiggins for advice and assistance. Richard Sailor provided us with translations of the papers by Akopyan et al. Seth Stein was supported by a fellowship from the Fannie and John Hertz Foundation. This research was also supported by National Science Foundation Grant EAR78-03653 at Stanford and National Science Foundation Grants EAR76-14262 and 7803653 at Stanford and by National Science Foundation Grant EAR77-14675 at Caltech.

\section{REFERENCES}

Akopyan, S., V. Zharkov, and V. Lyubimov (1975). The dynamic shear modulus in the interior of the earth (in Russian), Dok. Akad. Nauk SSSR 223, 1-3.

Akopyan, S., V. Zharkov, and V. Lyubimov (1976). Corrections to the eigenfrequencies of the earth due 
to the dynamic shear modulus (in Russian), Fiz. Zemli 12, 625-630.

Alsop, L. E., G. Sutton, and M. Ewing (1961a). Free oscillations of the earth observed on strain and pendulum seismographs, J. Geophys. Res. 66, 621-629.

Alsop, L. E., G. H. Sutton, and M. Ewing (1961b). Measurement of $Q$ for very long period free oscillations, J. Geophys. Res. 66, 2911-2915.

Anderson, D. L., and R. S. Hart (1976). An earth model based on free oscillations and body waves, $J$. Geophys. Res. 81, 1461-1475.

Backus, G. and F. Gilbert (1961). The rotational splitting of the free oscillations of the earth, Proc. Natl. Acad. Sci. U.S. 47, 362-371.

Benioff, H. (1959). Fused-quartz extensometer for secular, tidal and seismic strains, Bull. Geol. Soc. Am. 70, 1019-1032.

Benioff, H. (1960). Long period seismographs, Bull. Seism. Soc. Am. 50, 1-13.

Benioff, H., F. Press, and S. Smith (1961). Excitation of the free oscillations of the earth, J. Geophys. Res. 66, 605-619.

Buland, R. and F. Gilbert (1978). Improved resolution of complex eigenfrequencies in analytically continued spectra, Geophys. J. 52, 457-470.

Dahlen, F. A. (1968). The normal modes of a rotating elliptical earth, Geophys. J. 16, 329-367.

Geller, R. J. and S. Stein (1977). Split free oscillation amplitudes for the 1960 Chilean and 1964 Alaskan earthquakes, Bull. Seism. Soc. Am. 67, 651-660.

Gilbert, F. and G. Backus (1965). The rotational splitting of free oscillations of the Earth, 2, Rev. Geophys. 3, 1-9.

Kanamori, H. (1970). The Alaska earthquake of 1964: radiation of long-period surface waves and source mechanism, J. Geophys. Res. 75, 5029-5040.

Kanamori, H. and D. L. Anderson (1975). Amplitude of the earth's free oscillations and long-period characteristics of the earthquake source, J. Geophys. Res. 80, 1075-1078.

Kanamori, H. and J. J. Cipar (1974). Focal process of the great Chilean earthquake May 22, 1960, Phys. Earth Planet Interiors 9, 128-136.

Liu, H. P., D. L. Anderson, and H. Kanamori (1976). Velocity dispersion due to anelasticity: implications for seismology and mantle composition, Geophys. J. 47, 41-58.

Ness, N., J. Harrison, and L. Slichter (1961). Observations of the free oscillations of the earth, J. Geophys. Res. 66, 621-629.

Pekeris, C. L., Z. Alterman, and H. Jarosch (1961). Rotational multiplets in the spectrum of the earth, Phys. Rev. 122, 1692-1700.

Sailor, R. V. and A. M. Dziewonski (1978). Measurements and interpretation of normal mode attenuation, Geophys. J. 53, 559-581.

Saito, M. (1967). Excitation of free oscillations and surface waves by a point source in a vertically heterogeneous earth, J. Geophys. Res. 72, 3689-3699.

Slichter, L. B. (1967). Spherical oscillations of the earth, Geophys. J. 14, 171-177.

Smith, S. W. (1961). An investigation of the earth's free oscillations. Ph.D. Thesis, California Institute of Technology.

Stein, S. and R. J. Geller (1977). Amplitudes of the split normal modes of a rotating, elliptical earth excited by a double couple, J. Phys. Earth 25, 117-142.

Stein, S. and R. J. Geller (1978). Time-domain observations and synthesis of split spheroidal and torsional free oscillations of the 1960 Chilean earthquake: preliminary results, Bull. Seism. Soc. Am. 68, $325-332$.

Woodhouse, J. H. (1976). On Rayleigh's principle, Geophys. J. 46, 11-22.

Wiggins, R. A. and S. P. Miller (1972). New noise-reduction technique applied to long-period oscillations from the Alaskan earthquake, Bull. Seism. Soc. Am. 62, 471-479.

DEPARTMENT OF GEOPHYSICS

School of Earth SCIENCES

STANFORD UNIVERSITY

StanFord, California 94305

\author{
Seismological Laboratory \\ California Institute of Technology \\ Pasadena, California 91125 \\ Contribution No. 3159
}

Manuscript received May 26, 1978 